Highly Energetic Physical Processes and

Mechanisms for Emission from Astrophysical Plasmas

IAU Symposium, Vol. 195, 2000

P. C. H. Martens, S. Tsuruta, and M. A. Weber, eds.

\title{
Magnetohydrodynamic Turbulence in Accretion Discs
}

U. Torkelsson

Chalmers University of Technology/Göteborg University, Department of Theoretical Physics, Astrophysics Group, S-412 96 Gothenburg, Sweden

\author{
A. Brandenburg \\ Department of Mathematics, University of Newcastle upon Tyne, NE1 \\ YRU, U.K.
}
Å. Nordlund
Theoretical Astrophysics Center, Juliane Maries Vej 30, DK-2100
Copenhagen $\emptyset$, Denmark
R. F. Stein
Department of Physics and Astronomy, Michigan State University, East Lansing, MI 48824, U.S.A.

\begin{abstract}
We present results from numerical simulations of magnetohydrodynamic turbulence in accretion discs. Our simulations show that the turbulent stresses that drive the accretion are less stratified than the matter; thus, the surface layers are more strongly heated than the interior of the disc.
\end{abstract}

The most promising model for the angular momentum transport in accretion discs is based on the appearance of magnetohydrodynamic turbulence in Keplerian discs (e.g., Balbus \& Hawley 1998). Several groups have investigated the properties of this turbulence in numerical simulations (Hawley, Gammie, \& Balbus 1995; Matsumoto \& Tajima, 1995; Brandenburg et al. 1995; Stone et al. 1996). All of these simulations confirm the basic concept, that the turbulence works as a form of viscous friction that transports the angular momentum outwards and heats up the accretion disc. Recent simulations have extended the investigations to study the role of the turbulence in the response of the disc to a dynamic perturbation (Torkelsson et al. 2000), but in this paper we will limit ourselves to discussing the vertical distribution of the turbulent stresses.

We represent a small part of the accretion disc with a Cartesian box in which the Keplerian shear flow is linearized. The boundary conditions of the shearing box are (sliding) periodic in the (radial) azimuthal direction. The vertical boundaries are perfect conductors with respect to the magnetic field, and stress-free with no flow through the boundaries with respect to the velocity field.

The Shakura \& Sunyaev (1973) prescription for the turbulent viscosity predicts that the turbulent stresses should be proportional to the pressure. This is not what we find in our numerical simulations. In particular, we do not find 


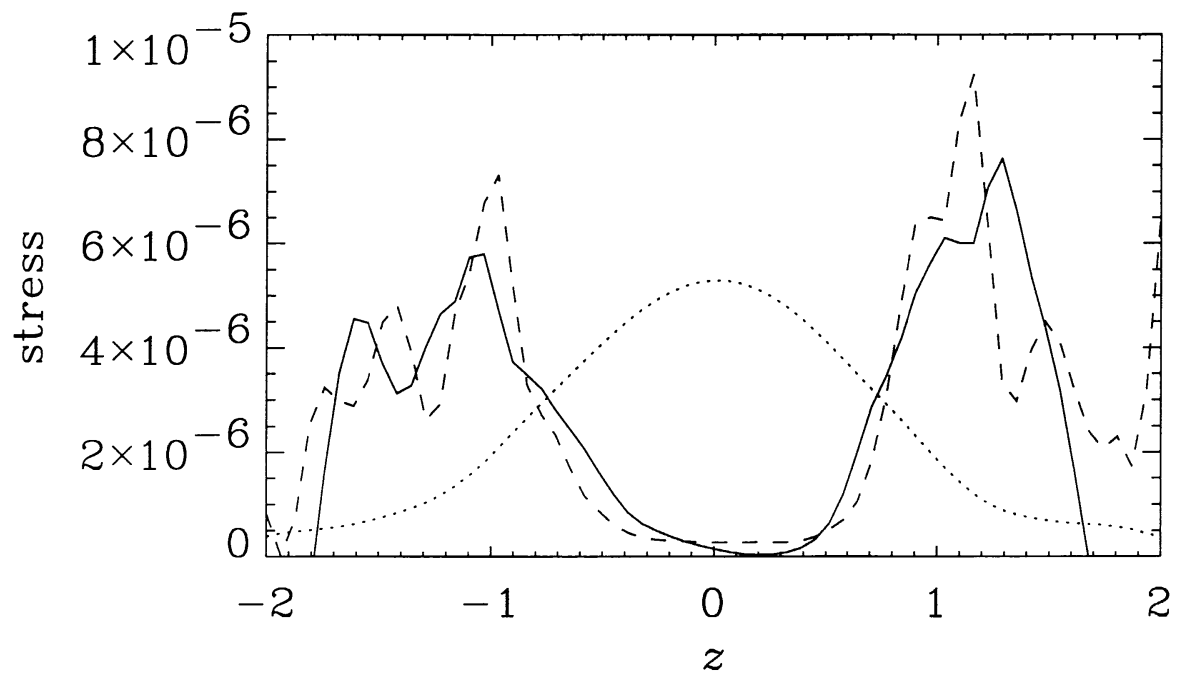

Figure 1. The turbulent stresses, $-B_{r} B_{\phi} / \mu_{0}$ (solid line) and $\rho u_{r} u_{\phi}$ (dashed line), as a function of $z$. As a comparison, we plot the pressure scaled down by a factor $10^{-2}$ (dotted line).

that the stresses decrease with increasing distance from the midplane of the disc as the pressure does (Figure 1).

Acknowledgments. Computer resources from the National Supercomputer Centre at Linköping University are gratefully acknowledged. UT is supported by the Natural Sciences Research Council (NFR), and RFS is supported by NASA grant NAG5-4031. This work was supported in part by the Danish National Research Foundation through its establishment of the Theoretical Astrophysics Center $(\AA N)$.

\section{References}

Balbus, S. A., \& Hawley, J. F. 1998, Rev. Mod. Phys., 70, 1

Brandenburg, A., Nordlund, Å., Stein, R. F., \& Torkelsson, U. 1995, ApJ, 446, 741

Hawley, J. F., Gammie, C. F., \& Balbus, S. A. 1995, ApJ, 440, 742

Matsumoto, R., \& Tajima, T. 1995, ApJ, 445, 767

Shakura, N. I., \& Sunyaev, R. A. 1973, A\&A, 24, 337

Stone, J. M., Hawley, J. F., Gammie, C. F., \& Balbus, S. A. 1996, ApJ, 463, 656

Torkelsson, U., Ogilvie, G. I., Brandenburg, A., Pringle, J. E., Nordlund, Å., \& Stein, R. F. 2000, MNRAS, submitted 